\title{
Clasificador de edad en imágenes digitales usando métodos estadísticos
}

\author{
Luis Enrique Colmenares Guillen ${ }^{1}$, Roberto Guillermo Torres López ${ }^{1}$, \\ Maya Carrillo Ruiz ${ }^{1}$, Francisco Javier Albores Velasco ${ }^{2}$ \\ ${ }^{1}$ Facultad de Ciencias de la Computación, Benemérita Universidad Autónoma de Puebla, \\ Puebla, México \\ ${ }^{2}$ Facultad de Ingeniería y Tecnología, Universidad Autónoma de Tlaxcala, Tlaxcala, México \\ \{lecolme,robert.torres.lopez, crrllrzmy, javier.albores\} @gmail.com
}

\begin{abstract}
Resumen. La estimación automática de edad tiene considerables aplicaciones en áreas como el marketing, como por ejemplo, al generar y mostrar contenido específico para cualquier grupo etario, y en la seguridad informática donde se permitiría proteger a menores de edad de contenidos no aptos para su edad. El objetivo general de este trabajo es mostrar la metodología desarrollada para generar un sistema clasificador basado en apariencia utilizando los modelos de representación: análisis de componentes principales y análisis discriminante lineal; y exponer los resultados obtenidos sobre las bases de imágenes FG-Net y IMDB-Wiki con las cuales se clasificaron en dos grupos, mayores $(+18)$ y menores (-18) de edad donde se obtuvo hasta un $89 \%$ de efectividad.
\end{abstract}

Palabras clave: FisherFaces, estimación de edad, análisis de componentes principales, análisis discriminante lineal, imágenes faciales, diferenciador de minoría de edad.

\section{Age Classifier in Digital Images Using Statistical Methods}

\begin{abstract}
The automatic age estimation has considerable applications in areas such as marketing, for example, while generating and displaying specific content for some age group, and in computing security, where protecting minors from contents not suitable for their age would be allowed. The general objective of this work is to show the methodology developed to generate a classifier system based on appearance, using representation models: analysis of main components and a linear discriminant analysis; and to present the results so obtained on the FG-Net and IMDB-Wiki images basis, with which were classified into two groups; major $(+18)$ and minor $(-18)$ of age, where up to an $89 \%$ of effectiveness was obtained.
\end{abstract}

Keywords: FisherFaces, age estimation, principal component analysis, linear discriminant analysis, facial images, age minority differentiator. 


\section{Introducción}

El reconocimiento de diferentes variaciones faciales como la expresión, el género o la identidad de una persona han sido extensamente estudiadas, en comparación con la estimación automática de edad que impacta directamente en áreas de marketing como indican Santos, Sánchez, Carmonet, Guerra y Casanova en [1], la seguridad informática de menores de edad sostiene Suárez Ricardo en [2] y en caso concreto permitirá al laboratorio de análisis forense de la Facultad de Ciencias de la Computación de la Benemérita Universidad Autónoma de Puebla implementar un detector de sitios web que almacenen y distribuyan contenido relacionado con pedofilia por ello se define el criterio de los grupos etarios de mayoría y minoría de edad.

Si bien, para los humanos es relativamente fácil clasificar a las personas en grupos de edad mediante rasgos faciales, para un computador no lo es debido a que el rostro humano es un objeto dinámico que tiene un alto grado de variabilidad y el proceso de envejecimiento de las caras es incontrolable y personalizado explican Nabil, Olwan, Tubeel, El-sar \& Sultan en [10].

\subsection{Modelos de representación de características}

En 1994 Kwon Young propuso por primera vez en [3] un modelo antropométrico donde la idea principal es consultar los estudios biológicos del desarrollo del cráneo que permite describir el crecimiento de la cabeza de un individuo desde su infancia hasta su edad adulta.

Así mismo, los puntos fiduciales permiten extraer de forma automática las características de un rostro que son registradas en un vector de 16 componentes que contiene relaciones entre distancias y áreas obtenidas entre los puntos marcados como demuestran Cox, Ghosn y Yianilos en [4].

Por último, Hayashi, Yasumoto e Ito en [5] utilizan los modelos de apariencia en los cuales se considera la textura de la piel y de la forma del rostro para caracterizar de forma adecuada cada una de las imágenes faciales. En esta publicación se realiza una estimación de edad por medio de un esquema de clasificación grupal usando una descripción semántica del rostro para la caracterización de los rasgos faciales.

\subsection{Algoritmos de estimación de edad}

La estimación de edad es un proceso de reconocimiento de patrones donde cada uno de los descriptores de edad es considerado una clase, por lo tanto, puede ser resuelta por medio de clasificadores por ejemplo el vecino más cercano como lo utiliza Khaung Tin en [11] o con redes neuronales demostrado por Hewahi, Olwan, Tubeel en [12] o puede abordarse como un problema de regresión donde la edad es un conjunto de valores secuenciales, se han desarrollado tres formulaciones para la función de envejecimiento: lineal, cuadrática y cubica. El modelo óptimo se obtuvo con imágenes faciales de diferentes edades basado en un algoritmo genético demostrado por Lanitis, Taylor, Cootes en [6]. 


\subsection{Métodos estadísticos como modelos de representación}

Los métodos estadísticos: análisis de componentes principales y análisis discriminante lineal son representados a través de los modelos de representación Eigenfaces y Fisherfaces.

La técnica de Eigenfaces fue propuesta por Turk y Pentland en [7] la cual consiste en encontrar los componentes principales de la distribución de rostros o los eigenvectores de la matriz de covarianza del conjunto de imágenes faciales, estos eigenvectores pueden ser utilizados como el conjunto de características que representan la variación entre las imágenes faciales.

Por otro lado, Belhumeur, Hespanha y Kriegman sostienen en [8] que las fisherfaces aumentan la relación entre la distribución de las distintas clases y la distribución intraclases.

\subsection{Análisis de componentes principales}

Es una técnica que toma una proyección lineal y maximiza la dispersión de todas las imágenes proyectadas. En primer lugar, se considera el conjunto de $\mathrm{N}$ imágenes con valores en el espacio de imágenes n-dimensional. Como lo indica la formula (1):

$$
\left\{x_{i}\right\} i=1,2, \ldots, N .
$$

Se asume además que cada una de las imágenes pertenece a una de las c clases $\left\{\mathrm{x}_{1}\right.$, $\left.\mathrm{x}_{2}, \ldots, \mathrm{x}_{\mathrm{c}}\right\}$ Asimismo, se considera una transformación lineal que lleva el espacio de imágenes original de $\mathrm{n}$ dimensiones al espacio de características de dimensión $\mathrm{m}$, donde $\mathrm{m}<\mathrm{n}$. Los nuevos vectores de características $\mathrm{Y}_{\mathrm{k}} \in \mathfrak{R}_{\mathrm{m}}$ son definidos por la siguiente transformación lineal, formula (2):

$$
\mathrm{Y}_{\mathrm{k}}=\mathrm{W}^{\mathrm{T}} \mathrm{X}_{\mathrm{k}} \quad \mathrm{K}=1,2, \ldots, \mathrm{N},
$$

donde $\mathrm{W} \in \mathfrak{R}^{\mathrm{nxm}}$ es una matriz con columnas ortonormales. Se define además la matriz de distribución total $\mathrm{S}_{\mathrm{T}}$ como la formula (3):

$$
\mathrm{S}_{\mathrm{T}}=\sum_{\mathrm{K}=1}^{\mathrm{N}}\left(\mathrm{x}_{\mathrm{k}}-\mu\right)\left(\mathrm{x}_{\mathrm{k}}-\mu\right)^{\mathrm{T}} .
$$

Donde $\mu \in \mathfrak{R}_{\mathrm{n}}$ es la media de todas las imágenes de (1). Luego de aplicar la transformación lineal $\mathrm{W}_{\mathrm{T}}$, la distribución de los vectores de características $\left\{\mathrm{y}_{1}, \mathrm{y}_{2}, \ldots\right.$, $\mathrm{y}_{\mathrm{N}}$ \} es $\mathrm{W}_{\mathrm{T}} \mathrm{S}_{\mathrm{Tw}}$. Se toma aquella proyección $\mathrm{W}_{\text {opt }}$ que maximiza el determinante de la distribución total de la matriz de las N imágenes. Como lo indica la formula (4):

$$
\mathrm{W}_{\text {opt }}=\arg \max \mathrm{W}\left|\mathrm{W}^{\mathrm{T}} \mathrm{S}_{\mathrm{T}} \mathrm{W}\right|=\left[\mathrm{w}_{1}, \mathrm{w}_{2} \ldots \mathrm{w}_{\mathrm{m}}\right] .
$$

\subsection{Análisis discriminante lineal}

Este método selecciona el $\mathrm{W}$ de la formula (2), de manera que el cociente entre la distribución de las diferentes clases y la distribución intra-clases sea máxima. Para esto, se define la matriz $\mathrm{S}_{\mathrm{B}}$ de distribución entre clases como lo indica la formula (5): 


$$
\mathrm{S}_{\mathrm{T}}=\sum_{\mathrm{i}=1}^{\mathrm{c}} \mathrm{N}_{\mathrm{i}}\left(\mu_{\mathrm{i}}-\mu\right)\left(\mu_{\mathrm{i}}-\mu\right)^{\mathrm{T}} .
$$

Y la matriz $S_{\mathrm{W}}$ de distribución intra-clases, como se puede apreciar en la formula (6).

$$
\mathrm{S}_{\mathrm{w}}=\sum_{\mathrm{i}=\mathrm{i}}^{\mathrm{c}} \sum_{\mathrm{x}_{\mathrm{k}} \in \mathrm{x}_{\mathrm{i}}} \mathrm{N}_{\mathrm{i}}\left(\mu_{\mathrm{i}}-\mu\right)\left(\mu_{\mathrm{i}}-\mu\right)^{\mathrm{T}},
$$

donde $\mu_{\mathrm{i}}$ es la imagen media de la clase $\mathrm{X}_{\mathrm{i}}, \mathrm{y} \mathrm{N}_{\mathrm{i}}$ es el número de imágenes en la clase $\mathrm{X}_{\mathrm{i}}$. Si la matriz $\mathrm{S}_{\mathrm{W}}$ es no singular, la proyección $\mathrm{W}_{\mathrm{opt}}$ se elige como la matriz con columnas ortonormales que aumenta el cociente del determinante de la matriz de distribución entre clases de las imágenes proyectadas y el determinante de la matriz de la distribución intra-clases de las $\mathrm{N}$ imágenes, de la formula (7):

$$
\mathrm{W}_{\text {opt }}=\arg \max \mathrm{W} \frac{\left|\mathrm{w}^{\mathrm{T}} \mathrm{S}_{\mathrm{B}} \mathrm{W}\right|}{\left|\mathrm{w}^{\mathrm{T}} \mathrm{S}_{\mathrm{w}} \mathrm{W}\right|}=\left[\mathrm{w}_{1}, \mathrm{w}_{2} \ldots \mathrm{w}_{\mathrm{m}}\right] \text {, }
$$

donde $\left\{\mathrm{W}_{\mathrm{i}} \mid \mathrm{i}=1,2, \ldots, \mathrm{m}\right\}$ es el conjunto de valores propios de $\mathrm{S}_{\mathrm{B}} \mathrm{y} \mathrm{S}_{\mathrm{W}}$ correspondiente a los m mayores valores propios $\left\{\lambda_{\mathrm{i}} \mid \mathrm{i}=1,2, \ldots, \mathrm{m}\right\}$, entonces, se muestra de la formula (8).

$$
\mathrm{S}_{\mathrm{B}} \mathrm{W}_{\mathrm{i}}=\lambda_{\mathrm{i}} \mathrm{S}_{\mathrm{W}} \mathrm{W}_{\mathrm{i}} \quad \mathrm{i}=1,2, \ldots, \mathrm{m} .
$$

Por lo tanto, se obtienen $\mathrm{c}-1$ valores propios distintos de cero, y por consiguiente el límite superior de m es $\mathrm{c}-1$, donde c es el número de clases, se muestra formula (9):

$$
\mathrm{W}_{\mathrm{pca}}^{\mathrm{T}}=\mathrm{W}_{\text {fld }}^{\mathrm{T}} \mathrm{W}_{\mathrm{pca}}^{\mathrm{T}},
$$

donde se derivan las formulas (10) y (11).

$$
\begin{gathered}
\mathrm{W}_{\mathrm{pca}}=\arg \max \mathrm{W}\left|\mathrm{W}^{\mathrm{T}} \mathrm{S}_{\mathrm{T}} \mathrm{W}\right|, \\
\mathrm{W}_{\text {fld }}=\arg \max \mathrm{W} \frac{\left|\mathrm{w}^{\mathrm{T}} \mathrm{w}_{\mathrm{pca}}^{\mathrm{T}} \mathrm{S}_{\mathrm{B}} \mathrm{W}_{\mathrm{pca}} \mathrm{W}\right|}{\mathrm{W}^{\mathrm{T}} \mathrm{w}_{\mathrm{pca}}^{\mathrm{T}} \mathrm{S}_{\mathrm{W}} \mathrm{W}_{\mathrm{pca}} \mathrm{W}} .
\end{gathered}
$$

\section{Trabajos relacionados}

Kwon y Lobo proponen en [9] un modelo antropométrico donde la idea principal es consultar los estudios biológicos del desarrollo cráneo facial existentes en la literatura. Esta teoría utiliza un modelo matemático de transformación craneal para describir el crecimiento de la cabeza de un individuo desde su infancia hasta su edad adulta.

El modelo de apariencia considera que la textura de la piel y de la forma del rostro pueden ser registrados en un vector de 16 o 71 componentes que contienen relaciones entre distancias y áreas obtenidas entre los puntos marcados como explican Cox el at. En [4] en ambas investigaciones el mayor problema es la incapacidad de estimar la edad en imágenes faciales que no estén completamente de frente. 
Finalmente, el modelo de apariencia considera que la textura de la piel y de la forma del rostro permiten caracterizar de forma adecuada cada una de las imágenes, usando una descripción semántica del rostro para caracterizar los rasgos faciales. Hayashi, Yasumoto e Ito en [5], usa este modelo con un esquema de clasificación grupal. Sanhueza Riveros en [10], genera un modelo clasificador con local binary pattern, buscando los elementos más significativos del rostro como son boca y ojos, en este trabajo se genera un clasificador de 4 diferentes grupos (niños, adolescentes, adultos y ancianos).

En [11] Khaung Tin, indica que la estimación de edad es vista como un proceso de reconocimiento de patrones donde cada uno de los descriptores actúa como una clase. De esta manera la estimación de edad se aprecia como un problema de clasificación resuelta con el algoritmo del vecino más cercano, así mismo, se hace referencia al análisis de componentes principales, como técnica estadística, y se hace un acercamiento a los Eigenfaces.

Las redes neuronales las utiliza Hewahi en [12], para clasificar en 4 grupos principales (niño (1-12), joven (13-25), adulto (26-45), viejo (46-63)) usando como conjuntos de entrenamiento las bases de datos de FG-NET la cual está compuesta por 1002 imágenes de diferentes sujetos de pruebas, con diferente iluminación y con un rango de edad de 0 a 62 años y empleando 4 capas de redes neuronales lograron obtener hasta un $78 \%$ de efectividad total con un porcentaje $87.6 \%, 67 \%, 86.4 \%$ y $70.9 \%$ respectivo a cada grupo principal.

Por otro lado, las edades son un conjunto de números con valores secuenciales, por lo tanto, la estimación de edad puede ser vista como un problema de regresión como lo explica Lanitis et al en [6], que propone un algoritmo genético usando funciones lineales, cuadráticas y cúbicas para representar el envejecimiento en las imágenes faciales.

En el tema de los modelos de apariencia se analizaron diversos trabajos que se relacionan entre sí siendo el punto de intersección la creación de los modelos con los métodos estadísticos ACP y ADL.

Terradez Gurrea indica en [13] que ACP es una técnica estadística de síntesis de la información o reducción del número de variables, es decir, ante un banco de datos con muchas variables, el objetivo será reducirlas a un menor número, perdiendo la menor cantidad de información posible.

Por otro lado, ADL maximiza la razón de dispersión entre clases a dispersión dentro de las clases. La idea es que las mismas clases deben de agruparse firmemente cerca, mientras que las clases diferentes están tan lejos como es posible en la representación de baja dimensión, como lo indica Hernandez y Mendez en [14].

Los métodos ACP y ADL, son estadísticos que funcionan para sintetizar la información y para maximizar la diferenciación entre clases respectivamente. En el ámbito de la visión por computadora ACP y ADL ofrecen una técnica llamada Fisherfaces, método propuesto por Belhumeur, Hespanha y Kriegman en [15].

De esta manera el modelo de apariencia permite obtener las características de las imágenes faciales generando los clasificadores con un conjunto de imágenes de entrenamiento con una descripción previa. 
En el artículo documental [16], Kyle Patterson presenta una propuesta para crear exhibidores interactivos para museos, los cuales mostrarán información variada para diferentes tipos de visitantes, en él se utilizan las Fisherfaces como método para obtener las características de las imágenes haciendo énfasis en la separación entre clases que genera este método.

Como resultado dio un porcentaje de efectividad del $72 \%$ en hombres y $62 \%$ en mujeres esto sin realizar algún preprocesamiento sobre las imágenes de entrenamiento o de prueba.

\section{Bases de datos y preprocesamiento}

\subsection{IMDB-Wiki data set}

Rothe, R. en [17] explica que IMBD-Wiki data set es una base de datos que extrae fotos de la página web IMDB y de Wikipedia, consiguiendo un total de 524,230 imágenes de rostros con información para obtener su edad. Se obtuvieron fotos de un rango de 0 a 100 años con variación de expresión, pose y luminosidad, ver figura 1.

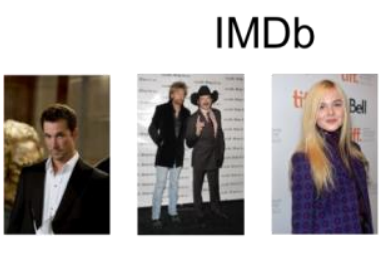

460,723 images
Wikipedia

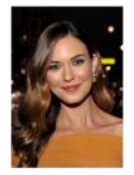

Fig. 1. Base de datos IMDB-WIKI.

\subsection{FG-Net}

FG-net base de datos de envejecimiento disponible en [18], es una base de imágenes pública que podemos utilizar para los experimentos. La base de datos contiene 1002 rostros a color y escala de grises de alta resolución con alta variación de luminosidad, pose expresión y tamaño. Contiene en total 82 sujetos de múltiples razas con un rango de edad de 0 a 69 años explicado por Guo, Fu y Dyer en [19] y es ampliamente usada como en [20] por Guo, Mu, Fu y Huang y por Luu, Ricanek, Bui y Suen en [21]. Ejemplo de imagen en FG-Net, figura 2.

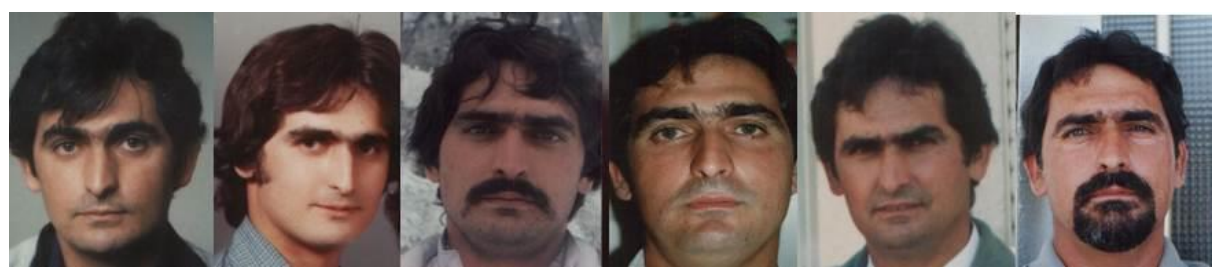

Fig. 2. Sujeto 13 Base de datos FG-Net. 


\subsection{Preprocesamiento}

Dado que la base de datos IMDB-wiki, data set no indica la edad de la persona que aparece en la foto se tiene que realizar un procedimiento para calcular la edad, esto es restar el año de nacimiento al año en que fue tomada la fotografía. Después de obtener las edades de cada imagen de la base de datos, enseguida se procede con el algoritmo para obtener el conjunto de imágenes con el que se entrenará el clasificador.

El siguiente paso de la metodología permite obtener los rostros de la base de imágenes con la única condición que la imagen sea mayor a 400x400 pixeles y eliminar la mayor cantidad de características de las imágenes que no aportan información al modelo, esto con el fin de optimizar y liberar carga computacional. Para ello se aplica el filtro escala de grises, se ecualiza el histograma y se extrae el área de interés para la estimación de edad, el resultado es una lista de imágenes de 200x200 pixeles con rostros ecualizados.

En la figura 3, se puede observar el preprocesamiento aplicado paso a paso a un elemento de la base de imágenes FG-net.

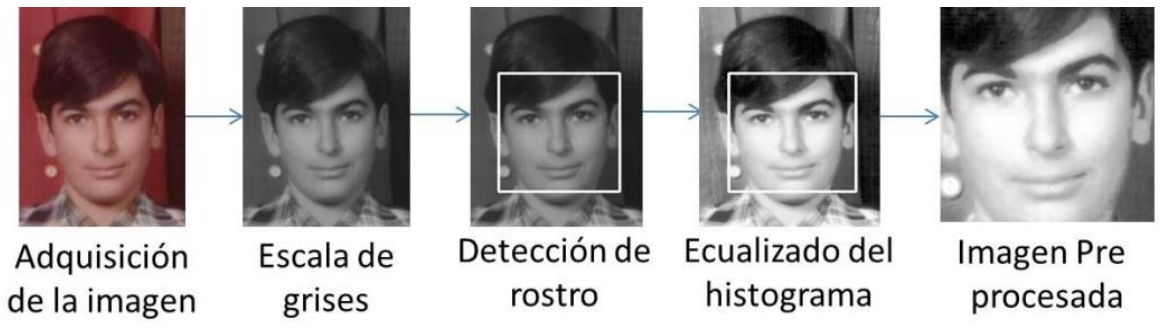

Fig. 3. Preprocesamiento de las imágenes de entrenamiento.

\section{Propuesta}

En este trabajo se propone el desarrollo de un clasificador basado en apariencia, de acuerdo en lo documentado por Suárez Ricardo en [2], Kyle Patterson en [16] y Sanhueza Riveros en [10] debido que este tipo de clasificadores obtienen buenos resultados sin importar que los rostros varíen en pose y luminosidad siendo este un problema recurrente cuando se emplean puntos fiduciales como lo demuestran Cox el al. en [4] o clasificadores basados en modelos antropométricos trabajados por Kwon y Lobo en [9] donde se tiene que preseleccionar las imágenes ocupando solamente las que contienen rostros completamente frontales.

En los trabajos de investigación el clasificador funciona mediante dos partes específicas, la creación de los modelos de representación de características y la clasificación de la nueva imagen. En la figura 4 se observa la propuesta del clasificador. 
Creación de los modelos

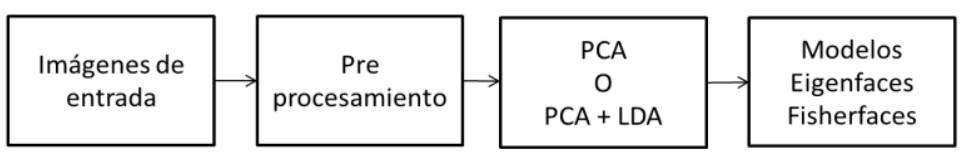

Estimación de la nueva imagen

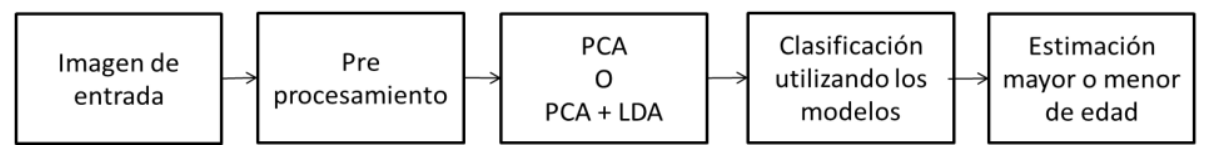

Fig. 4. Propuesta del clasificador.

\section{Metodología}

Como se indica la figura 4, el clasificador está conformado por dos módulos separados, el primero está orientado a la creación de los modelos que describan las características de los conjuntos de imágenes de cada clase haciendo énfasis en sus diferencias, para generar los modelos se utiliza el conjunto de imágenes preprocesadas resultado del procedimiento anterior y se asigna una cantidad similar de imágenes para cada clase después se aplica sobre ellas los métodos estadísticos: Análisis de Componentes Principales (Eigenfaces) usado por Turk et al. en [7] (figura 5) o la combinación de Análisis de Componentes Principales y Análisis Discriminante Lineal (Fisherfaces) introducido por Belhumeur et al. en [8] (figura 6).

La segunda parte corresponde a la clasificación de las nuevas imágenes, para poder clasificarlas se debe usar el mismo preprocesamiento, filtro de escala de grises, ecualizado del histograma y obtener el área de interés. Una vez que se obtiene la imagen preprocesada se extraen las características con el mismo método estadístico (ACP o la combinación ACP y ADL) con el que se haya entrenado el clasificador. Por último, se utiliza el clasificar K-nn para verificar con que clase se tiene un mayor número de características semejantes, como se desarrolla en la figura 7.

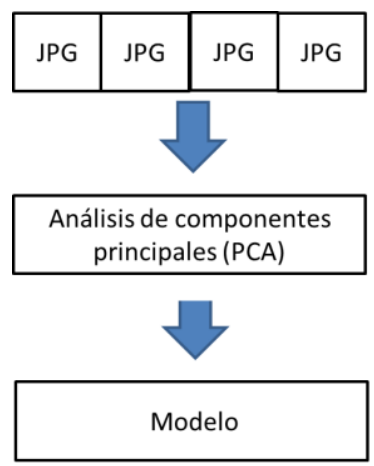

Fig. 5. Creación de modelos Eigenfaces. 


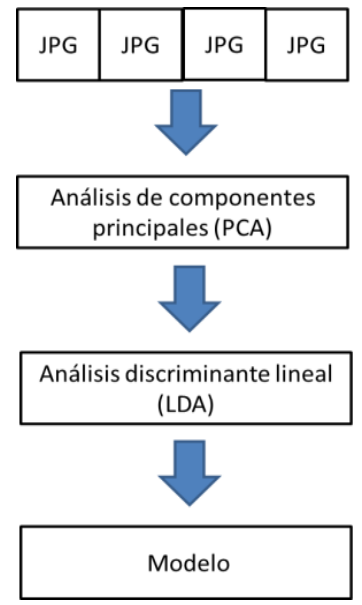

Fig. 6. Creación de modelos Fisherfaces.

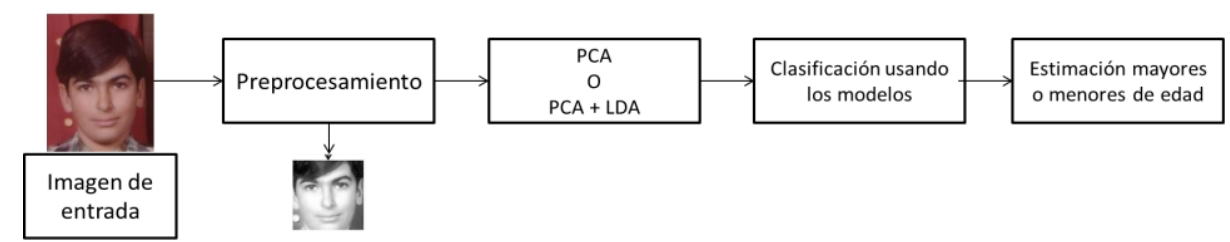

Fig. 7. Proceso de clasificación de una nueva imagen.

El modelo general del clasificador se compone de tres modelos independientes que son adaptados como se describe en la figura 8, en los siguientes pasos.

1.- Preprocesamiento de las imágenes; Permite cargar un conjunto de imágenes y mediante un proceso que incluye aplicación de filtros (Escala de grises y ecualizado del histograma) y detección y extracción de rostros mediante la técnica Haar-like features se obtiene una lista de imágenes faciales depurada y estandarizada.

2- Modelo de representación de características; Toma las imágenes preprocesadas y realiza un Análisis de Componentes Principales o una combinación de la misma técnica seguida de un Análisis Discriminante Lineal, este proceso genera los modelos de representación estadística de las imágenes haciendo énfasis en las diferencias entre clases.

3.- Clasificación de la nueva imagen; Una vez que se crearon y cargaron los modelos de representación, se comparan con una nueva imagen de prueba la cual es preprocesada con los mismos filtros y se extraen sus características usando los mismos filtros y el mismo modelo de representación que en las imágenes de entrenamiento con el fin de obtener la estimación aproximada del grupo etario al que pertenece el nuevo rostro.

La implementación de la metodología se realizó en una computadora personal acer aspire modelo E5-411 con un procesador Intel Celeron N2830, 4GB DDR3 Memoria Ram y 1000GB de Disco Duro. 
Luis Enrique Colmenares Guillen, Roberto Guillermo Torres López, Maya Carrillo Ruiz, et al.

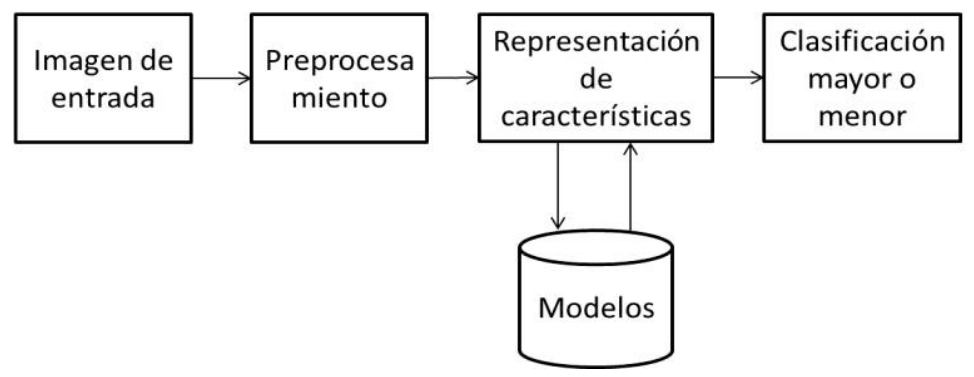

Fig. 8. Modelo general.

\section{Resultados}

La exactitud que tiene un clasificador se mide mediante el número de aciertos que realiza en función del número de pruebas que se llevan a cabo. Sin embargo, un sistema que acierte siempre puede asignar gran cantidad de falsos positivos, y por el contrario, un clasificador estricto que minimice los falsos positivos puede llegar a aumentar el número de falsos negativos. Es por ello que la evaluación de un clasificador deberá de considerar todos los aspectos anteriores, y proporcionar una medida que evalúe simultáneamente la capacidad de discernir a qué clase pertenece cada dato. Por lo tanto, se utilizan tres criterios basados en la matriz de confusión (Figura 9) que son precisión, recall y F-measure, Factor $\mathrm{F}$ (F - Measure), que es la media armónica entre Precisión (P) y Recall (R).

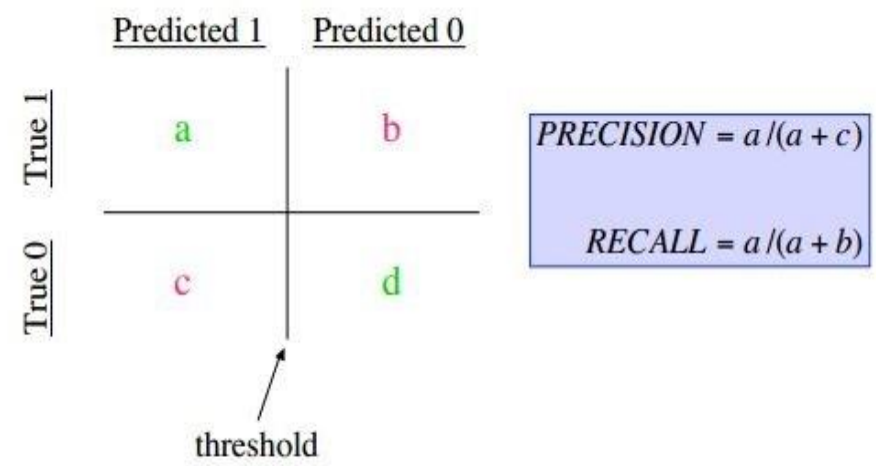

Fig. 9. Matriz de confusion (Performance Measures for Machine Learning [22]).

Para la primera parte del experimento se utilizaron las imágenes de entrenamiento de la base de datos FG-Net generando dos clases representativas, mayores $(+18)$ y menores $(-18)$ de edad de las cuales se obtuvieron los siguientes resultados, que se muestran en la tabla 1 y en la tabla 2 . 
Tabla 1. Resultados con la base de datos Fg-Net y Eigenfaces.

\begin{tabular}{|c|c|c|c|c|}
\hline Imágenes & Efectividad & Precisión & Recall & $\mathrm{F}$ \\
\hline 10 & $40 \%$ & 0.70 & 0.56 & 0.68 \\
\hline 50 & $55 \%$ & 0.86 & 0.56 & 0.68 \\
\hline 90 & $62 \%$ & 0.90 & 0.62 & 0.74 \\
\hline 120 & $64 \%$ & 0.91 & 0.63 & 0.75 \\
\hline 150 & $78 \%$ & 0.91 & 0.65 & 0.77 \\
\hline
\end{tabular}

Tabla 2. Resultados con la base de datos Fg-Net y Fisherfaces.

\begin{tabular}{|c|c|c|c|c|}
\hline Imágenes & Efectividad & Precisión & Recall & $\mathrm{F}$ \\
\hline 10 & $43 \%$ & 0.79 & 0.46 & 0.58 \\
\hline 50 & $60 \%$ & 0.88 & 0.62 & 0.71 \\
\hline 90 & $64 \%$ & 0.91 & 0.63 & 0.75 \\
\hline 120 & $70 \%$ & 0.87 & 0.76 & 0.81 \\
\hline 150 & $71 \%$ & 0.89 & 0.73 & 0.81 \\
\hline
\end{tabular}

Para la segunda parte del experimento se utilizó un total de 2000 imágenes seleccionadas de IMDB-Wiki data set y se aplicó sobre ellas el mismo preprocesamiento con el objetivo de construyeron los modelos de las mismas clases representativas del experimento anterior, mayores (+18) y menores (-18) de edad obteniendo los resultados mostrados en la tabla 3 y en la tabla 4.

Tabla 3. Resultados con la base de datos IMDB-Wiki y Eigenfaces.

\begin{tabular}{|c|c|c|c|c|}
\hline Imágenes & Efectividad & Precisión & Recall & $\mathrm{F}$ \\
\hline 2000 & $77 \%$ & 0.92 & 0.80 & 0.83 \\
\hline
\end{tabular}

Tabla 4. Resultados con la base de datos IMDB-Wiki y Fisherfaces.

\begin{tabular}{|c|c|c|c|c|}
\hline Imágenes & Efectividad & Precisión & Recall & F \\
\hline 2000 & $89 \%$ & 0.97 & 0.89 & 0.86 \\
\hline
\end{tabular}

En la tabla 5, se muestran los porcentajes en cuanto a efectividad en la clasificación de edad, resultado del presente trabajo junto con los obtenidos por otros autores que ocupan diferentes metodologías. En la figura 10, se muestra, un resultado positivo del clasificador con un menor y un mayor de edad. 


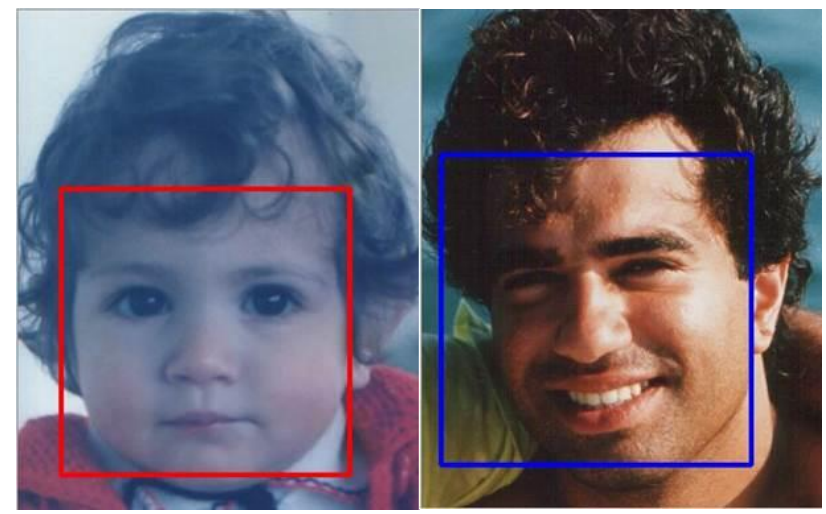

Fig. 10. Resultado del clasificador (rojo menor de edad, azul mayor de edad).

Tabla 5. Comparación de mejores resultados en efectividad con trabajos relacionados.

\begin{tabular}{|c|c|c|c|c|}
\hline $\begin{array}{c}\text { Eigenfaces } \\
\text { propio }\end{array}$ & $\begin{array}{c}\text { Fisherfaces } \\
\text { propio }\end{array}$ & $\begin{array}{c}\text { Modelo } \\
\text { antropométrico } \\
{[9]}\end{array}$ & $\begin{array}{c}\text { Redes } \\
\text { Neuronales } \\
{[12]}\end{array}$ & $\begin{array}{c}\text { Eigenfaces } \\
{[16]}\end{array}$ \\
\hline $77 \%$ & $89 \%$ & $92 \%$ & $86 \%$ & $72 \%$ \\
\hline
\end{tabular}

\section{Discusión}

La estimación de edad automática de edad más específicamente la clasificación de mayores y menores de edad es un problema que impacta en distintas áreas con múltiples aplicaciones potenciales, por ello se desarrolló un clasificador que puede realizar esta tarea. Según los resultados encontrados en esta investigación y en comparación con los previamente investigados en el estado del arte podemos concluir que nuestro clasificador obtiene buenos resultados con imágenes provenientes de entornos reales (cámara web, fotogramas de video y fotografías en entornos no controlados) a diferencia de los clasificadores basados en modelos antropométricos y puntos fiduciales los cuales obtienen mejores resultados con imágenes donde el rostro se encuentra completamente de frente, aunque no se puede clasificar imágenes con variación de pose, que está demostrado en el trabajo por Kwon et al. en [9], por Cox et al. en [4] y Hewahi et al. en [12].

También se observó que existen investigaciones de clasificadores basados en apariencia que obtienen mejores resultados debido a que realizan un preprocesamiento más exhaustivo como Kyle Patterson en [16], que realiza un etiquetado de las imágenes por grupo etario y sexo y Kwon Young en [3] el cual obtiene todos los elementos característicos del rostro lo cual representa un alto costo computacional. Otro punto a resaltar del presente trabajo es la demostración de la precisión del clasificador que aumenta a medida del número de imágenes de entrenamiento como lo afirman Santos et al. en [1] y Suárez Ricardo en [2]. Por último se recomienda el uso de la base de datos FG-net la cual es ampliamente usada en investigaciones como las realizadas por 
Luu et al. en [21], Guo et al. en [20] y Hewahi et al. en [12], debido a que proporciona un conjunto de imágenes de entrenamiento y de prueba con su respectiva edad.

\section{Conclusiones y trabajo a futuro}

En este trabajo se desarrolló un clasificador basado en apariencia utilizando dos modelos diferentes de representación, Eigenfaces y Fisherfaces. Se demostró que las pruebas realizadas con cada modelo, el porcentaje de aciertos es proporcional al número de imágenes utilizadas para su creación, debido a que se extraen y clasifican más características en cada clase. Esto indica que si se tiene mayor número de imágenes se obtienen mejores resultados de estimación. Esto se demuestra al verificar que los mejores resultados se obtienen cuando se utilizaron 2000 imágenes para la creación de los modelos donde se obtuvo una efectividad del $89 \%$ en la diferenciación de mayoría o minoría de edad.

También se determinó que el clasificador desarrollado con Fisherfaces, presenta mejores resultados comparado al clasificador basado en Eigenfaces. Por último, se muestra que el número de imágenes incide directamente sobre el tamaño de los modelos estadísticos.

El trabajo a futuro corresponde a la recolección de nuevas bases de imágenes faciales que brinden la edad de los individuos. Así mismo el generar una nueva metodología basada en multiprocesamiento y procesamiento con GPU que permita trabajar con grandes volúmenes de imágenes sin que esto conlleve a un problema de rendimiento.

\section{Referencias}

1. Santos, S., Sanchez, A., Carmonet, B., Guerra, C., Santos, S.: Control de edad en redes sociales mediante biometría facial (2012)

2. Suárez-Fuentes, R. A.: Diferenciación visual de niños y adultos a partir de imágenes faciales (2013)

3. Kwon, Y.: Age classification from facial images in Computer Vision and Pattern Recognition. In: IEEE Computer Society Conference, pp. 762-767 (1994)

4. Cox, I., Ghosn, J., Yianilos, P.: Features based face recognition using mixture-distance in Computer Vision and Pattern Recognition. In: IEEE computer Society Conference, pp. 209-216 (1996)

5. Hayashi, J., Yasumoto, M., Ito, H., Koshimizu, H.: Method for estimating and modeling age and gender using facial image processing. In: Virtual Systems and Multimedia, IEEE Proceedings, Seventh International Conference, pp. 439-448 (2001)

6. Lanitis, A., Taylor, C., Cootes, T.: Toward automatic simulation of aging effects on face images. In: Transactions on Pattern Analysis and Machine Intelligence. 24(4), pp. 442-455 (2002)

7. Turk, M., Pentland, A.: Eigenfaces for recognition. Journal of Cognitive Neuroscience (1991) 
8. Belhumeur, P., Hespanha, J., Kriegman, D.: Eigenfaces vs fisherfaces: Recognition using class specific linear projection. In: Bernard F. Buston and Roberto Cipolla. Springer, Vol. 1064, pp. 45-58 (1996)

9. Kwon, Y., Vitoria, L.: Age classification from facial images. Computer Vision and Image Understanding, 74(1), pp. 1-21 (1999)

10. Sanhueza, R.: Análisis y detección de características faciales usando aprendizaje estadístico. Tesis del Universidad de Chile, Facultad de ciencias Físicas y Matemáticas (2008)

11. Tin, H.: Gender and Age Estimation Based on Facial Images. Act technical Napocensis, 52(3), pp. 37 (2011)

12. Hewahi, N., Olwan, A., Tubeel, N., El-Asar, S., Abusultan, Z.: Age estimation based on neural networks using face features. Journal of Emerging Trends in Computing and Information Sciences, 1(2), pp. 61-67 (2010)

13. Terradez, G.: Análisis de componentes principales. Universidad Oberta de Cataluña, 11 (2002)

14. Hernández, J., Méndez, N.: Reconocimiento de expresiones faciales para interacción con el computador. Jóvenes en la ciencia, 2(1), pp. 62-66 (2016)

15. Belhumeur, P., Hespanha, J., Kriegman, D.: Eigenfaces vs fisherfaces: Recognition using class specific linear projection. Transactions on pattern analysis and machine intelligence, 19(7), pp. 711-720 (1997)

16. Patterson, K.: Automatic Age Estimation and Interactive Museum Exhibits. Experimental Aging Research, 35(2), pp. 268-275 (2014)

17. Rothe, R., Timofte, R., Van, G.: Dex: Deep proceeding. In: Proceedings of the IEEE. International Conference on Computer Vision Workshops, pp. 10-15 (2015)

18. FG-Net. Disponible en: http://www.fgnet.rsunit.com

19. Gou, G., Gu, Y., Dyer, C., Huang, T.: Image-based human age estimation by manifold learning and locally adjusted robust regression. IEEE Transactions on Image Processing, 17(7), pp. 1178-1188 (2008)

20. Gou, G., Gu, Y., Huang, T.: Human age estimation using bio-inspired features. In: Computer vision and Pattern Recognition. IEEE conference, pp. 112-119 (2009)

21. Luu, K., Ricanek, K., Bui, T., Suen, C.: Age estimation using active appearance models and support vector machine regression. In: Biometrics: Theory, Applications and Systems. IEEE 3rd International Conference, pp. 1-6 (2009)

22. Performance Measures for Machine Learning. Disponible en: https://www.cs.cornell. edu/courses /cs578/2003fa/performance_measures.pdf) 